\title{
Exact Travelling Wave Solutions of the Nonlinear Evolution Equations by Improved F-Expansion in Mathematical Physics
}

\author{
Md. Habibul Bashar ${ }^{1 *}$, Md. Mamunur Roshid ${ }^{2}$
}

\begin{abstract}
With the assistance of representative calculation programming, the present paper examines the careful voyaging wave arrangements from the general $(2+1)$-dimensional nonlinear development conditions by utilizing the Improved F-expansion strategy. As a result, the used technique is effectively utilized and recently delivered some definite voyaging wave arrangements. The recently created arrangements have been communicated as far as trigonometric and hyperbolic capacities. The created arrangements have been returned into their relating condition with the guide of emblematic calculation programming Maple. Among the produced solutions, some solutions have been visualized by $3 \mathrm{D}$ and $2 \mathrm{D}$ line graphs under the choice of suitable arbitrary parameters to show their physical interpretation. The delivered arrangements show the intensity of the executed technique to evaluate the accurate arrangements of the nonlinear (2+1)-dimensional nonlinear advancement conditions, which are reasonably pertinent for using nonlinear science, scientific material science and designing. The Improved F-expansion method is a reliable treatment for searching essential nonlinear waves that enrich a variety of dynamic models that arise in engineering fields.
\end{abstract}

Keywords: The Improved F-expansion scheme, The general (2+1)-dimensional nonlinear evolution equation, Traveling wave solutions, Soliton solution.

2010 AMS: Primary 35C07, 35C08

${ }^{1}$ Department of Mathematics, European University of Bangladesh, Dhaka, Bangladesh, ORCID: https://orcid.org/0000-0002-0734-1487.

${ }^{2}$ Department of Mathematics, Pabna University of Science and Technology, Pabna, Bangladesh.

*Corresponding author: hbashar27.1273@gmail.com and habibul.bashar@eub.edu.bd

Received: 13 December 2019, Accepted: 1 July 2020, Available online: 29 September 2020

\section{Introduction}

As of late, nonlinear incomplete differential conditions (NPDEs) is comprehensively used to delineate various huge marvels and dynamic methodology in various fields of science and designing, particularly in liquid mechanics, hydrodynamics, numerical science, dissemination process, strong state material science, plasma material science, neural material science, substance energy and geo-optical filaments. In this article, we will study the generalized (2+1)-dimensional nonlinear evolution equations in the form

$$
u_{x t}+u_{x x y}+a u_{x} u_{x y}+b u_{x x} u_{y}=0
$$

Recently, some special cases of Eq. (1.1) have been studied by several authors[1]-[4]. When setting a $=4$ and $b=2$, we get:

$$
u_{x t}+u_{x x x y}+4 u_{x} u_{x y}+2 u_{x x} u_{y}=0
$$


Eq. (1.2) known as the Calogero-Bogoyavlenskii-Schiff (CBS) equation with (2+1) dimensional.

where $u=u(x, y, t)$ is used for brevity. In the article, subscript occurring with a term denotes its partial derivative with respect to the subscript variable.in equ.(1.2) $u_{t}$ describes the time evolution of the wave, while the terms $u_{x x} u_{y}$ and $u_{x} u_{x y}$ are account for nonlinearity of the wave. The CBS equation has some physical situated history like it very well may be composed as potential structure [5]. The CBS equation was at first built by Bogoyavlenskii and Schiff in different ways [6]-[9]. The ongoing history of some past inquires about show that few powerful strategies for getting definite arrangements of the CBS condition are contributed by a differing gathering of specialists over the globe [10]-[13], for instance, the periodic and soliton solutions of the CBS equation were gotten by Gandarias et al.[10]. Its integrability has been demonstrated by Zhang et al.[11] and derived also the symmetry reductions of the equation. Li and Chen [12] found the exact solutions by using the generalized Raccati equation expansion method. Wang and Yang [13] employed the Hirota Bilinear strategy for construction of the quasi-periodic wave solutions in terms of theta functions for a Hirota bilinear equation.

When setting $a=-4$ and $b=-2$, we get:

$$
u_{x t}+u_{x x x y}-4 u_{x} u_{x y}-2 u_{x x} u_{y}=0
$$

Eq. (3) known as the breaking soliton equation with $(2+1)$ dimensional.

When setting $\mathrm{a}=4$ and $\mathrm{b}=4$, we get:

$$
u_{x t}+u_{x x y}+4 u_{x} u_{x y}+4 u_{x x} u_{y}=0
$$

Eq. (4) known as the Bogoyavlenskii equation with $(2+1)$ dimensional.

Numerous researchers arranged through nonlinear evolution equations (NEEs) to build voyaging wave arrangement by executing a few techniques. The methods that are entrenched in ongoing writing, for example, the extended Kudryashov method[14], the modified simple equation method [15], the new extended $\left(G^{\prime} / G\right)$ expansion method [16]-[17], the Darboux transformation [18], the trial solution method [19], the Exp-Function Method [20], the multiple simplest equation method [21], $\exp (-\phi(\xi))$-expansion method [22]-[26], Pseudo parabolic model [27]-[29], Sine-Gordon expansion method[30], Complex solitons in the conformable (2+1)-dimensional Ablowitz-Kaup-Newell-Segur equation [31], Modified auxiliary expansion method [32], Method of line [33], Bernoulli sub-equation function method [34]-[35], The modified exponential function method [36], Improved Bernoulli sub equation function method [37], Finite difference method [38] and so on.

The target of this article is to apply the Improved F-expansion technique to build the precise voyaging wave answers for nonlinear advancement conditions in scientific material science by means of the generalized (2+1)-dimensional nonlinear evolution equations.

The article is set up as pursues: In section 2, the Improved F-expansion scheme has been talked about. In segment 3, we apply this plan to the nonlinear development conditions raised previously. In section 4, represents Results and Discussion, In section and in section 5 ends are given.

\section{Description of the Improved F-Expansion Method}

In this segment, we portray in subtleties The Improved F-extension strategy technique for discovering traveling wave equations of nonlinear equations. Any nonlinear condition in two free factors $x$ and $t$ can be communicated in following structure:

$$
\operatorname{Re}\left(u, u_{t}, u_{x}, u_{t t}, u_{x x}, u_{x t} \ldots \ldots \ldots\right)=0
$$

where, $u(\xi)=u(x, t)$ is an unknown function, $\Re$ is a polynomial of $u(\xi)=u(x, t)$ and its partial derivatives in which the highest order derivatives and nonlinear terms are included.

Step 1: The given PDE (2.1) can be changed into ODE utilizing the change $\xi=x \pm \omega t$ where $\omega$ is the speed of traveling wave such that $\omega \in R-\{0\}$

The traveling wave change grants us to diminish Eq. (2.1) to the following ODE:

$$
\Re\left(u, u^{\prime}, u^{\prime \prime}, \ldots \ldots \ldots \ldots \ldots . . . . . .\right)=0
$$

where $\Re$ is a polynomial in $u(\xi)$ and its derivatives, where $u^{\prime}(\xi)=\frac{d u}{d \xi}, u^{\prime \prime}(\xi)=\frac{d^{2} u}{d \xi^{2}}$, and so on.

step 2:Suppose the solution of Eq. (2.2) can be expressed by a polynomial in $\psi(\xi)$ :

$$
U=u(\xi)=\sum_{j=0}^{N} \alpha_{j}(\psi(\xi))^{j}+\sum_{j=1}^{N} \beta_{j}(\psi(\xi))^{-j}
$$


where

$$
\psi(\xi)=(m+\varphi(\xi))
$$

Here $\alpha_{N}$ or $\beta_{N}$ may be zero, but both could not be zero simultaneously. $\alpha_{j}(j=0,1,2 \cdots N), \beta_{j}(j=1,2 \cdots N)$ and $m$ are constants to be determined later and $\Phi(\xi)$ satisfies the ODE in form:

$$
\varphi^{\prime}(\xi)=K+\varphi^{2}(\xi)
$$

We now present three cases of the general solutions of the Riccati Equation (2.5) (Cruz, Schuch, Castanos and Rosas-Ortiz, 2015).

Case-I: When $K<0$, we get following hyperbolic solution

$$
\begin{aligned}
& \Phi(\xi)=-\sqrt{-K} \tanh (\sqrt{-K} \xi) \\
& \Phi(\xi)=-\sqrt{-K} \operatorname{coth}(\sqrt{-K} \xi)
\end{aligned}
$$

Case-II: When $K>0$, we get following trigonometric solution

$$
\begin{aligned}
& \Phi(\xi)=\sqrt{K} \tan (\sqrt{K} \xi) \\
& \Phi(\xi)=-\sqrt{K} \cot (\sqrt{K} \xi)
\end{aligned}
$$

Case-III: When $K=0$, we get following solution

$$
\Phi(\xi)=-\frac{1}{\xi}
$$

Step 3: The value of the positive integer $N$ can be determined by balancing the highest order linear terms with nonlinear terms of the highest order appearing in Eq. (2.2).

If the degree of $u(\xi)$ is $D[u(\xi)]=n$, then the degree of the other expressions will be as follows:

$$
D\left[\frac{d^{p} u(\xi)}{d \xi^{p}}\right]=n+p, \quad D\left[u^{p}\left(\frac{d^{q} u(\xi)}{d \xi q}\right)^{s}\right]=n p+s(n+q)
$$

Step 4: Substituting Eq. (2.3) along with Eqs. (2.4) and (2.5) into Eq. (2.2), we obtain polynomials in $(m+\varphi(\xi))^{j}$ and $(m+\varphi(\xi))^{-j},(j=0,1,2, \cdots, N)$. Gathering every coefficient of the came about polynomials to zero, yields an over-decided arrangement of arithmetical equations for $\alpha_{j}, \beta_{j}, \omega$ and $m$.

Step 5: Assume the estimation of the constants can be gotten by fathoming the mathematical conditions got in step 4 . Substituting the estimations of the constants together with the arrangements of Eq. (2.5), we will acquire new and far reaching precise traveling wave arrangements of the nonlinear development Eq. (2.1).

\section{Application of the Method}

In this section, we will exert the Improved F-expansion method to solve the equation (1.1).Now Using the traveling wave variable $\xi=x+y-\omega t$ and integrating with respect to $\xi$ reduces Eq. (1.1) to the following ordinary differential equation for $u=u(\xi)$.

$$
-\omega u^{\prime}+\left(\frac{a+b}{2}\right)\left(u^{\prime}\right)^{2}+u^{\prime \prime \prime}=0
$$

Where, primes denote the differentiation with regard to $\xi$ By balancing $u^{\prime \prime \prime}$ and $\left(u^{\prime}\right)^{2}$ we obtain $N=1$.Therefore the Improve F-expansion method admits to solution of (2.1) in the form

$$
U(x, y, t)=\alpha_{0}+\alpha_{1}(m+\varphi(\xi))+\beta_{1}(m+\varphi(\xi))^{-1}
$$

Now, substituting Eq. (3.2) into Eq. (3.1), and equating the coefficients of the powers $\varphi(\xi)$ then we obtain a system of algebraic equations. Solving this system of equations for $\alpha_{0}, \alpha_{1}, \beta_{1}, m$ and $\omega$ we obtain the following set values: 
Set-1: $m=m, \omega=-4 K, \alpha_{0}=\alpha_{0}, \alpha_{1}=0, \beta_{1}=\frac{12\left(m^{2}+K\right)}{a+b}$.

Set-2: $m=m, \omega=-4 K, \alpha_{0}=\alpha_{0}, \alpha_{1}=-\frac{12}{a+b}, \beta_{1}=0$.

Set-3: $m=0, \omega=-16 K, \alpha_{0}=\alpha_{0}, \alpha_{1}=-\frac{12}{a+b}, \beta_{1}=\frac{12 K}{a+b}$.

Case-I: When $K<0$, we get following hyperbolic solution

Family-1

$$
\begin{aligned}
& U_{1}(x, y, t)=\alpha_{0}+\frac{12\left(m^{2}+K\right)}{(a+b)(m-\sqrt{-K} \tanh (\sqrt{-K} \xi))} \\
& U_{2}(x, y, t)=\alpha_{0}+\frac{12\left(m^{2}+K\right)}{(a+b)(m-\sqrt{-K} \operatorname{coth}(\sqrt{-K} \xi))}
\end{aligned}
$$

where, $\omega=-4 K$ and $\xi=x+y-\omega t$.

Family-2

$$
\begin{aligned}
& U_{3}(x, y, t)=\alpha_{0}-\frac{12(m-\sqrt{-K} \tanh (\sqrt{-K} \xi))}{(a+b)} \\
& U_{4}(x, y, t)=\alpha_{0}-\frac{12(m-\sqrt{-K} \operatorname{coth}(\sqrt{-K} \xi))}{(a+b)}
\end{aligned}
$$

where, $\omega=-4 K$ and $\xi=x+y-\omega t$.

Family-3

$$
\begin{aligned}
& U_{5}(x, y, t)=\alpha_{0}+\frac{12 \sqrt{-K} \tanh (\sqrt{-K} \xi)}{(a+b)}-\frac{12 K}{(a+b) \sqrt{-K} \tanh (\sqrt{-K} \xi)} \\
& U_{6}(x, y, t)=\alpha_{0}+\frac{12 \sqrt{-K} \operatorname{coth}(\sqrt{-K} \xi)}{(a+b)}-\frac{12 K}{(a+b) \sqrt{-K} \operatorname{coth}(\sqrt{-K} \xi)}
\end{aligned}
$$

where, $\omega=-16 K$ and $\xi=x+y-\omega t$.

Case-II: When $K>0$, we get following trigonometric solution

Family-4

$$
\begin{aligned}
& U_{7}(x, y, t)=\alpha_{0}+\frac{12\left(m^{2}+K\right)}{(a+b)(m+\sqrt{K} \tan (\sqrt{K} \xi))} \\
& U_{8}(x, y, t)=\alpha_{0}+\frac{12\left(m^{2}+K\right)}{(a+b)(m-\sqrt{K} \cot (\sqrt{K} \xi))}
\end{aligned}
$$

where, $\omega=-4 K$ and $\xi=x+y-\omega t$.

Family-5

$$
\begin{aligned}
& U_{9}(x, y, t)=\alpha_{0}-\frac{12(m+\sqrt{K} \tan (\sqrt{K} \xi))}{(a+b)} \\
& U_{10}(x, y, t)=\alpha_{0}-\frac{12(m-\sqrt{K} \cot (\sqrt{K} \xi))}{(a+b)}
\end{aligned}
$$

where, $\omega=-4 K$ and $\xi=x+y-\omega t$.

Family-6

$$
\begin{aligned}
& U_{11}(x, y, t)=\alpha_{0}+\frac{12 \sqrt{K} \tan (\sqrt{K} \xi)}{(a+b)}+\frac{12 K}{(a+b) \sqrt{K} \tan (\sqrt{K} \xi)} \\
& U_{12}(x, y, t)=\alpha_{0}+\frac{12 \sqrt{K} \cot (\sqrt{K} \xi)}{(a+b)}-\frac{12 K}{(a+b) \sqrt{K} \cot (\sqrt{K} \xi)}
\end{aligned}
$$


where, $\omega=-16 K$ and $\xi=x+y-\omega t$.

Case-III: When $K=0$, we get following rational solution

Family-7

$$
U_{13}(x, y, t)=\alpha_{0}+\frac{12\left(m^{2}+K\right)}{(a+b)\left(m-\frac{1}{\xi}\right)}
$$

where, $\omega=-4 K$ and $\xi=x+y-\omega t$.

Family-8

$$
U_{14}(x, y, t)=\alpha_{0}-\frac{12\left(m-\frac{1}{\xi}\right)}{(a+b)}
$$

where, $\omega=-4 K$ and $\xi=x+y-\omega t$.

Family-9

$$
U_{15}(x, y, t)=\alpha_{0}+\frac{12}{(a+b) \xi}-\frac{12 K \xi}{(a+b)}
$$

where, $\omega=-16 K$ and $\xi=x+y-\omega t$.

\section{Results and Discussion}

Around there, we will discuss the physical depiction of the procured careful and singular wave answer for the general $(2+1)$-dimensional nonlinear advancement condition. We address these arrangements in graphical and check about the kind of arrangement. Presently we pictorial some get arrangements acknowledge by applied techniques for the general (2+1)-dimensional nonlinear advancement condition.
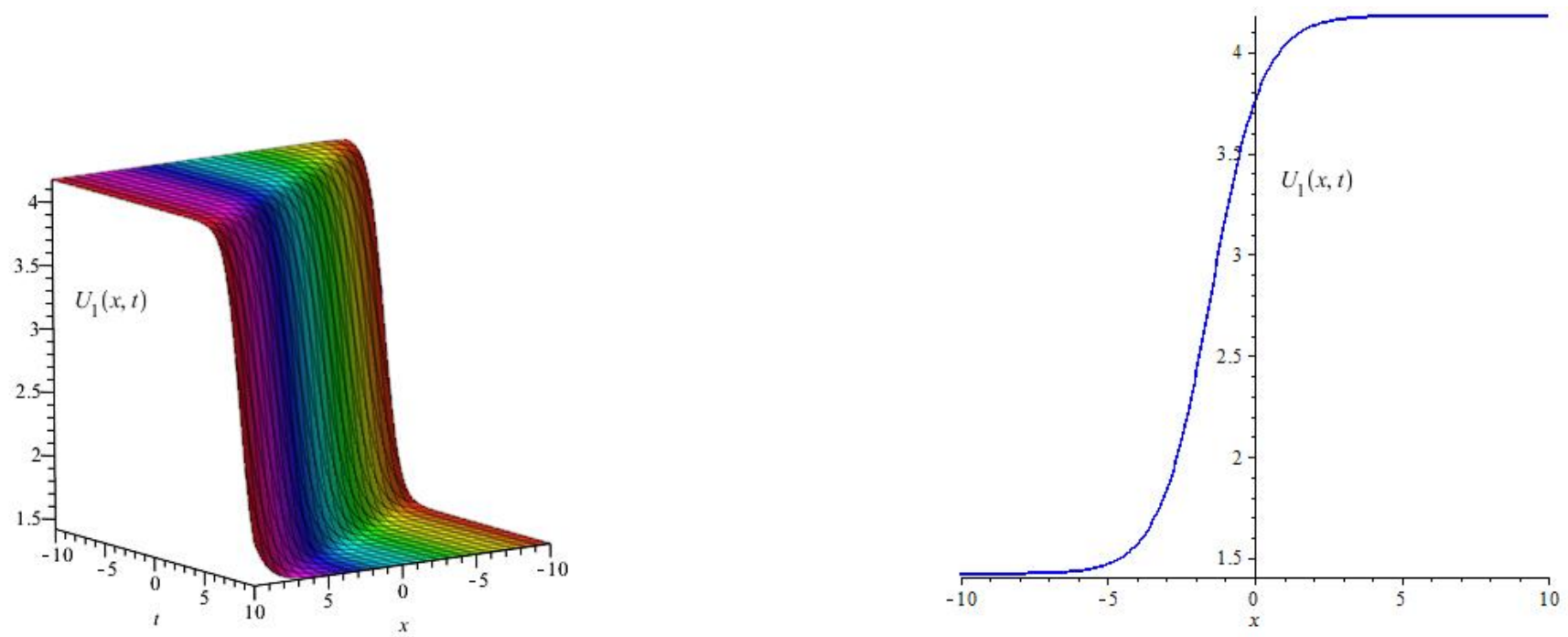

Figure 4.1. Kink Shape of $U_{1}(\xi)$ for $a_{0}=-2, a=2, b=3, m=2, K=-.33, y=2$ within $-10 \leq x, t \leq 10$. The left-sided figure shows the 3D plot and the right-sided figure shows the 2D plot for $t=0$ 

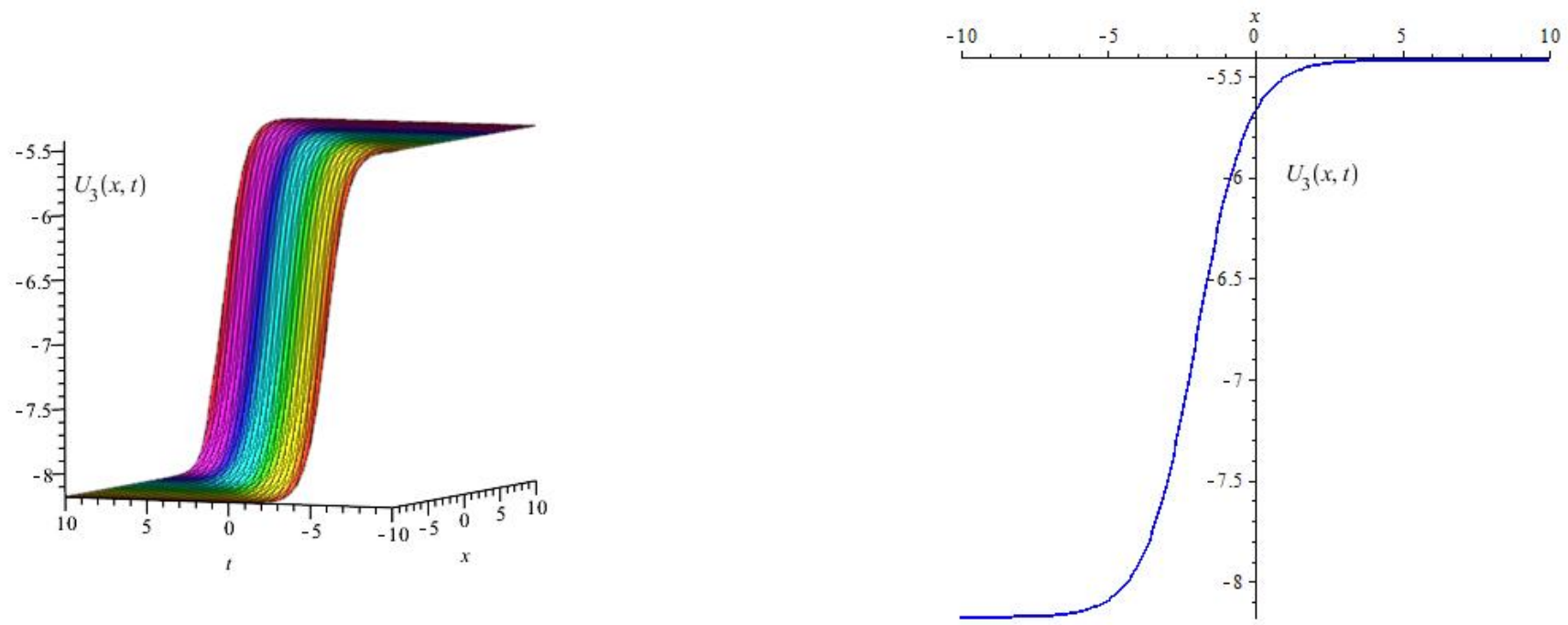

Figure 4.2. Kink Shape of $U_{3}(\xi)$ for $a_{0}=-2, a=2, b=3, m=2, K=-.33, y=2$ within $-10 \leq x, t \leq 10$. The left-sided figure shows the $3 \mathrm{D}$ plot and the right-sided figure shows the $2 \mathrm{D}$ plot for $t=0$

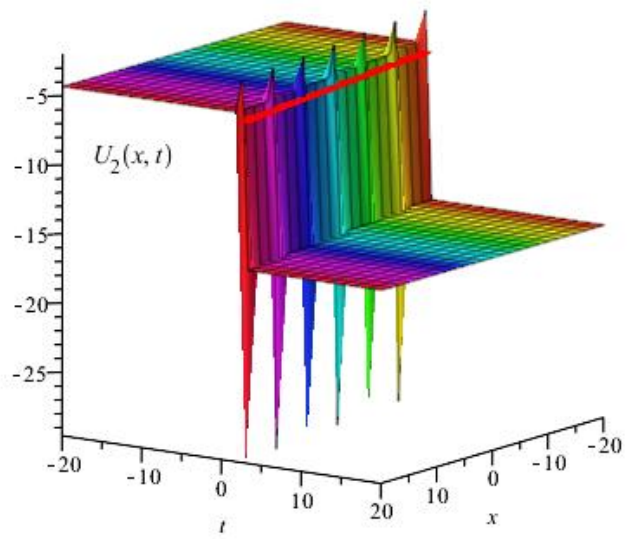

Figure 4.3. Kink Shape of $U_{2}(\xi)$ for $a_{0}=-2, a=2, b=1, m=-2, K=-2, y=0$ within $-10 \leq x, t \leq 10$. 

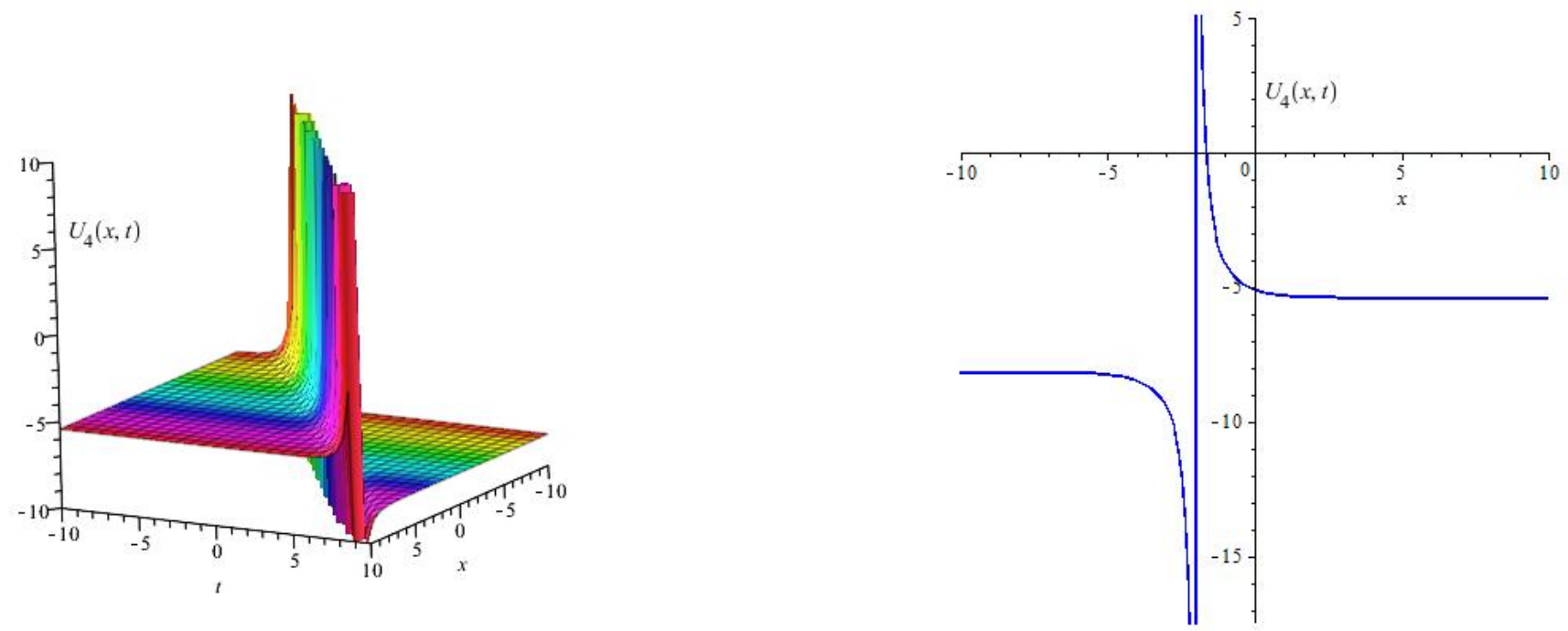

Figure 4.4. Singular Kink Shape of $U_{4}(\xi)$ for $a_{0}=-2, a=2, b=3, m=2, K=-.33, y=2$ within $-10 \leq x, t \leq 10$. The left-sided figure shows the 3D plot and the right-sided figure shows the 2D plot for $t=0$
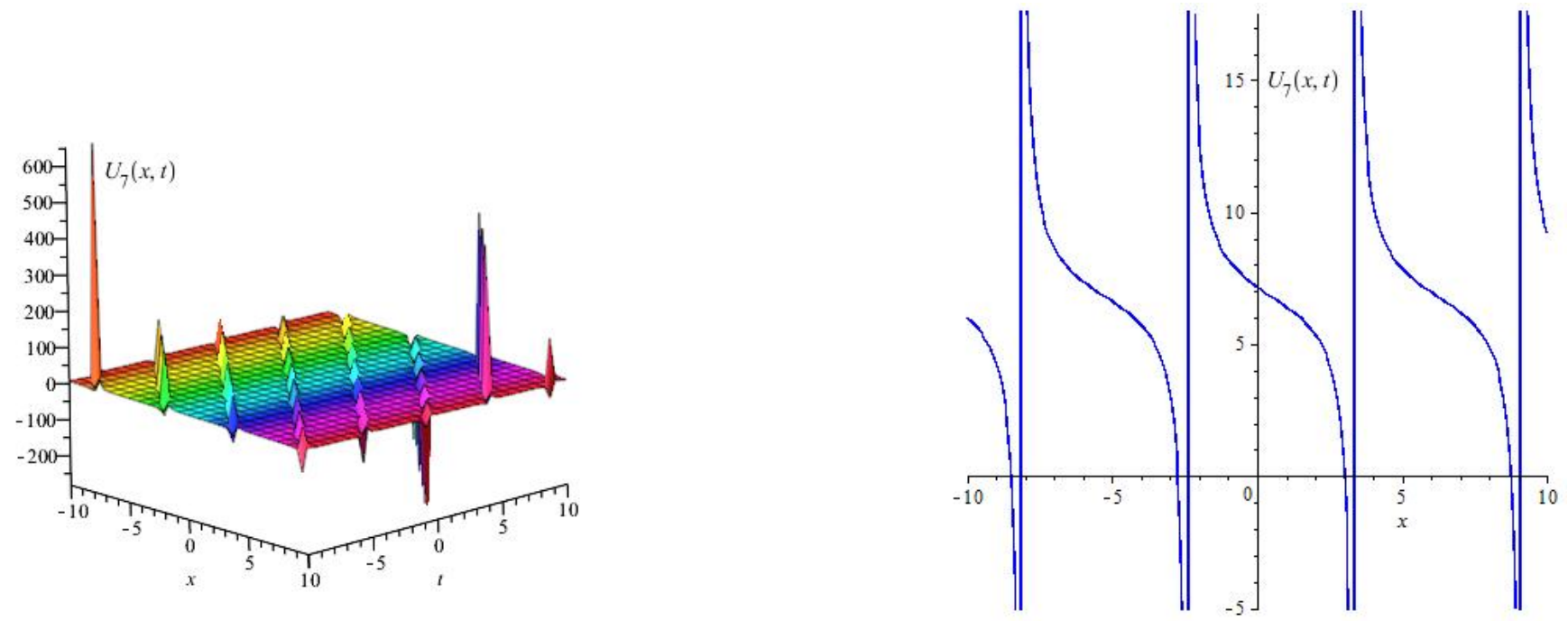

Figure 4.5. Periodic $\mathrm{N}$ soliton Shape of $U_{7}(\xi)$ for $a_{0}=2, a=2, b=3, m=2, K=.3, y=0$ within $-10 \leq x, t \leq 10$. The left-sided figure shows the 3D plot and the right-sided figure shows the 2D plot for $t=0$

\section{Conclusion}

In this segment, we have seen that two kinds of traveling wave arrangements as far as hyperbolic and trigonometric capacities for the general $(2+1)$-dimensional nonlinear evolution equation is effectively discovered by utilizing the Improved F-expansion method. From our outcomes got in this paper, we finish up the Improved F-expansion scheme strategy is amazing, powerful and helpful. The exhibition of this technique is dependable, basic and gives numerous new arrangements. Likewise, the arrangements of the proposed nonlinear development conditions in this paper have numerous potential applications in atomic and molecule material science. At long last, this technique gives a ground-breaking scientific instrument to get increasingly broad accurate arrangements of a large number of nonlinear PDEs in numerical material science.

\section{References}

[1] M.O. Al-Amr, Exact solutions of the generalized (2+1)-dimensional nonlinear evolution equations via the modified simple equation method, Comput. Math. Appl, 69(5) (2015), 390-397. 
[2] M. Najafi, S. Arbabi, New Exact Solutions for the Generalized (2+1)-dimensional Nonlinear Evolution Equations by Tanh-Coth Method,. Int. J. Modern Theoretical Phys., 2(2) (2013), 79-85.

[3] M. Najafi, S. Arbabi, M. Najafi, New application of sine-cosine method for the generalized (2+1) dimensional nonlinear evolution equations, Int. J. Adv. Math. Sci.,1(2) (2013), 45-49.

[4] M. Darvishi, M. Najafi, M. Najafi, New application of EHTA for the generalized (2+1)- dimensional nonlinear evolution equations, Int. J. Math. Comp. Sci., 6(3) (2010), 132-138.

[5] O. I. Bogoyavlenskii, Overturning solitons in new two-dimensional integrable equations, Mathematics of the USSRIzvestiya, 34(2) (1990), 245-259.

[6] A. M. Wazwaz, New solutions of distinct physical structures to high-dimensional nonlinear evolution equations, Applied Mathematics and Computation, 196 (2008), 363-370.

[7] Y. Peng, New types of localized coherent structures in the Bogoyavlenskii-Schiff equation,Int. J. Theor. Phys., 45(9) (2006), 1779-1783.

[8] T. Kobayashi, K. Toda, The Painleve test and reducibility to the canonical forms for higher-dimensional soliton equations with variable-coefficients, Symmetry, Inerrability and Geometry,Methods and Applications, 2 (2006),1-10.

[9] M. S. Bruzon, M. L. Gandarias, C. Muriel, J. Rami rez, S. Saez, F. R. Romero, The Calogero-Bogoyavlenskii-Schiff equation in (2+1)-dimensions, Theoretical and Mathematical Physics, 137(1) (2003), 1367-1377.

[10] M. L. Gandarias, M. S. Bruzon, Symmetry group analysis and similarity solutions of the CBS equation in (2+1)-dimensions, Proceedings of Applied Mathematics and Mechanics, 8 (2008), 10591-10592, DOI10 . 1002 /pamm. 200810591

[11] H. P. Zhang, Y. Chen, B. Li, Infinitely many symmetries and symmetry reduction of the (2+1)dimensional generalized Calogero-Bogoyavlenskii-Schiff equation, Acta Physica Sinaca, 58 (2009), 7393-7396.

[12] B. Li, Y. Chen, Exact analytical solutions of the generalized Calogero-Bogoyavlenskii-Schiff equation using symbolic computation, Czechoslovak Journal of Physics, 54 (2004), 517-528.

[13] J. Wang, X. Yang, Quasi-periodic wave solutions for the $(2+1)$-dimensional generalized Calogero-Bogoyavlenskii-Schiff (CBS) equation, Nonlinear Analysis, 75 (2012), 2256-2261.

[14] E. Yasar, Y. Yıldırım, A. Adem, Perturbed optical solitons with spatio-temporal dispersion in $(2+1)$-dimensions by extended Kudryashov method,Optik, 158 (2018), 1-14.

[15] H. Roshid, M. Roshid, N. Rahman, M. R. Pervin, New solitary wave in shallow water, plasma and ion acoustic plasma via the GZK-BBM equation and the RLW equation,Propulsion and Power Research, 6(1) (2017), 49-57.

[16] H. Roshid, M. A. Hoque, M. A. Akbar, New extended (G'/G)-expansion method for traveling wave solutions of nonlinear partial differential equations (NPDEs) in mathematical physics,Italian. J. Pure Appl. Math., 33 (2014), 175-190.

[17] L. Feng, T. Zhang, Breather wave, rogue wave and solitary wave solutions of a coupled nonlinear Schrodinger equation,Appl. Math. Lett., 78(2018), 133-140.

[18] X. Shuwei, H. Jingsong,The rogue wave and breather solution of the Gerdjikov-Ivanov equation,J. Math. Phys. 53 (2012).

[19] A. Biswas, M. Mirzazadeh, M. Eslami, Q. Zhou, A. Bhrawy, M. Belic,. Optical solitons in nano-fibers with spatio-temporal dispersion by trial solution method, Optik, 127(18) (2016), 7250-7257.

[20] J. Heris, I. Zamanpour,Analytical treatment of the Coupled Higgs Equation and the Maccari System via Exp-Function Method,Acta Universitatis Apulensis, 33 (2013), 203-216.

[21] Y. Zhao,New Exact Solutions for a Higher-Order Wave Equation of KdV Type Using the Multiple Simplest Equation Method,Journal of Applied Mathematics,(2014), 1-13.

[22] M. Islam, H. Roshid,Application of -expansion method for Tzitzeica type nonlinear evolution equations,Journal for Foundations and Applications of Physics, 4 (1) (2017).

[23] N. Rahman, S. Akter,H. Roshid,M. Alam,Traveling Wave Solutions of The (1+1)-Dimensional Compound KdVB equation by $\exp (-\phi(\xi))$-Expansion Method,Global Journal of Science Frontier Research, 13 (8) (2014), 7-13.

[24] R. Islam, M. Alam, A. Hossain, H. Roshid, M. Akbar, Traveling wave solutions of nonlinear evolution equations via $\exp (-\phi(\xi))$-Expansion Method,Global Journal of Scientific Frontier Research, 13 (11) (2014), 63-71.

[25] N. Kadkhoda, H. Jafari,Analytical solutions of the Gerdjikov-Ivanov equation by using $\exp (-\phi(\xi))-\operatorname{expansion}$ method,Optik, 139 (2017), 72-76. 
[26] B. Amfilokhiev, I. Voitkunskii, P. Mazaeva, S. Khodorkovskii,Flows of polymer solutions in the case of convective accelerations, Tr. Leningr. Korablestroit. Inst., 96 (1975),3-9.

[27] M. Roshid,H. Roshid, Exact and explicit traveling wave solutions to two nonlinear evolution equations which describe incompressible viscoelastic Kelvin-Voigt fluid,Heliyon, 4 (2018).

[28] O. Gozuk1z1l, S. Akcag1l, The tanh-coth method for some nonlinear pseudoparabolic equations with exact solutions,Advances in Difference Equations, 143 (2013).

[29] A. Turgut, T. Aydemir, A. Saha, A. Kara, Propagation of nonlinear shock waves for the generalised Oskolkov equation and its dynamic motions in the presence of an external periodic perturbation,Pramana - J. Phys., (2018), 78-90.

[30] J.L.G Guirao .,H. M. Baskonus, A. Kumar, M.S. Rawat, G. Yel, Complex Patterns to the (3+1)-Dimensional B-type Kadomtsev-Petviashvili-Boussinesq Equation, Symmetry, 12(1) (2020).

[31] W. Gao, G. Yel,H. M. Baskonus, C. Cattani, Complex solitons in the conformable (2+1)-dimensional Ablowitz-KaupNewell-Segur equation, Aims Math., 5(1) (2020), 507-521.

[32] W. Gao, H. F. Ismael, H. Bulut, .H. M Baskonus, Instability modulation for the (2+1)-dimension paraxial wave equation and its new optical soliton solutions in Kerr media, Physica Scripta, (2019),DOI : 10 . 1088 / $1402-4896$ / ab 4 a 50.

[33] W. Gao, M. Partohaghighi, H. M. Baskonus, S. Ghavi, Regarding the group preserving scheme and method of line to the numerical simulations of Klein-Gordon model,Results Phys., 15 (102555) (2019), 1-7.

[34] H. M. Baskonus, S. Ghavi, New singular soliton solutions to the longitudinal wave equation in a magneto-electro-elastic circular rod with MM-derivative,Modern Phys. Letters B, 33(21) (2019), 1950251-(1-16).

[35] H. M. Baskonus, Complex Soliton Solutions to the Gilson-Pickering Model, Axioms, 8(1) (2019), 18.

[36] O.A. Ilhan, A. Esen, H. Bulut, H. M. Baskonus, Singular solitons in the pseudo-parabolic model arising in nonlinear surface waves, Results Phys , 12 (2019), 1712-1715.

[37] G. Yel, H. M. Baskonus, H. Bulut, Regarding some novel exponential travelling wave solutions to the Wu-Zhang system arising in nonlinear water wave model, Indian J. Phys., 93(8) (2019), 1031-1039.

[38] A. Yokus, Numerical solution for space and time fractional order Burger type equation, Alexandria Eng. J. 57(3) (2018), 2085-2091. 